\section{(C) OPEN ACCESS}

\title{
Integrative network and brain expression analysis reveals mechanistic modules in ataxia
}

\author{
Ilse Eidhof, ${ }^{\oplus 1}$ Bart P van de Warrenburg, ${ }^{2}$ Annette Schenck ${ }^{\oplus 1}$
}

- Additional material is published online only. To view, please visit the journal online (http://dx.doi.org/10.1136/ jmedgenet-2018-105703).

'Department of Human Genetics, Donders Institute for Brain, Cognition, and Behavior, Radboud University Medical Centre, Nijmegen, The Netherlands

${ }^{2}$ Department of Neurology, Donders Institute for Brain, Cognition, and Behavior, Radboud University Medical Centre, Nijmegen, The Netherlands

\section{Correspondence to} Dr Annette Schenck, Department of Human Genetics, Donders Institute for Brain, Cognition, and Behavior, Radboud University Medical Centre, Nijmegen 6525GC, The Netherlands;

Annette.Schenck@radboudumc. $\mathrm{nl}$ and $\mathrm{Dr}$ Bart $\mathrm{P}$ van de Warrenburg, Department of Neurology, Donders Institute for Brain, Cognition, and Behavior, Radboud university medical centre, Nijmegen 6525GA, The Netherlands;

Bart.vandeWarrenburg@ radboudumc.nl

Received 22 August 2018 Revised 14 November 2018 Accepted 30 November 2018 Published Online First 27 December 2018

\begin{abstract}
Background Genetic forms of ataxia are a heterogenous group of degenerative diseases of the cerebellum. Many causative genes have been identified. We aimed to systematically investigate these genes to better understand ataxia pathophysiology.

Methods A manually curated catalogue of 71 genes involved in disorders with progressive ataxias as a major clinical feature was subjected to an integrated gene ontology, protein network and brain gene expression profiling analysis.
\end{abstract}

Results We found that genes mutated in ataxias operate in networks with significantly enriched protein connectivity, demonstrating coherence on a global level, independent of inheritance mode. Moreover, elevated expression specifically in the cerebellum predisposes to ataxia. Genes expressed in this pattern are significantly over-represented among genes mutated in ataxia and are enriched for ion homeostasis/synaptic functions. The majority of genes mutated in ataxia, however, does not show elevated cerebellar expression that could account for region-specific degeneration. For these, we identified defective cellular stress responses as a major common biological theme, suggesting that the defence pathways against stress are more critical to maintain cerebellar integrity than integrity of other brain regions. Approximately half of the genes mutated in ataxia, mostly part of the stress module, show higher expression at embryonic stages, which argues for a developmental predisposition.

Conclusion Genetic defects in ataxia predominantly affect neuronal homeostasis, to which the cerebellum appears to be excessively susceptible. Based on the identified modules, it is conceivable to propose common therapeutic interventions that target deregulated calcium and reactive oxygen species levels, or mechanisms that can decrease the harmful downstream effects of these deleterious insults.

\section{INTRODUCTION}

Genetic cerebellar ataxias are a group of disabling disorders that share progressive incoordination of movement due to dysfunction and degeneration of the cerebellum as their main hallmark. ${ }^{1}$ The advent of next-generation sequencing technologies greatly advanced the identification of genes involved in ataxia. ${ }^{2}$ However, despite the increasing number of genes identified, treatment attempts are still limited to relieving symptoms and do not target the underlying biological mechanisms. Development of effective therapies is hampered by an enormous genetic heterogeneity, the rarity of some the subtypes, and the limited knowledge of biological processes in which ataxia genes exert their function. Identification of shared biological modules between genes mutated in ataxia (from here onwards referred to as ataxia genes) would provide a basis for therapeutic strategies that could be applied to larger cohorts of ataxia patients, in spite of their heterogeneous genotypic background. In recent years, efforts have been made to identify common denominators of genetic ataxias. A number of ataxia genes were found to share interaction partners at protein level and to be involved in processes such as RNA splicing, regulation of transcription, and cell cycle. $^{2-5}$ When impaired in animal models, these processes lead to neurodegeneration, suggesting that these shared biological pathways maintain the integrity of the cerebellum and its connections. ${ }^{3}$ Nevertheless, previous studies focused primarily on protein networks among specific ataxia genes and subtypes, ${ }^{2-5}$ and only very few probed the influence of gene expression on cerebellar pathology. ${ }^{4}$

In this study, we systematically analyse the genes to date implicated in cerebellar ataxia, their functional biological pathways and their expression in the developing human brain. Our integrative study identifies common denominators that underlie progressive cerebellar degeneration and ataxia, including a cerebellum-specific mechanism affected in a subgroup of ataxia disorders that may account for region-specific degeneration and defective stress defence pathways as underlying mechanism to the large majority of ataxia disorders to which the cerebellum is in particular sensitive.

\section{MATERIALS AND METHODS \\ Cerebellar ataxia gene selection and classification}

The Human Phenotype Ontology database ${ }^{6}$ (download November 2015) was used to search for genes associated with ataxia. A list of 347 genes was obtained (online supplementary table S1) and manually curated using PubMed and OMIM. Genes associated with progressive cerebellar ataxia as prominent clinical manifestation, either in isolation or as part of a more complex phenotype, were included. Primary metabolic disorders, genes associated with cerebellar hypoplasia and genes inconsistently associated with cerebellar ataxia were excluded.

\section{Gene ontology analysis}

The webtool G-profiler ${ }^{7}$ (rev 1760, build October 2018) was used to perform gene ontology 
(GO) analysis of four categories of ataxia genes (all genes, dominant genes, recessive genes, polyglutamine (polyQ) genes; the latter refers to the group of dominant ataxias genes that, when mutated, carry a coding CAG repeat expansion that leads to polyQ expansion in the protein). For this study, we only considered GO terms (Biological Process, Molecular Function and Cellular Component) that were significantly enriched after correction for multiple testing (Bonferroni test, $\mathrm{p}<0.05$ ). GO analysis for the developmental transcriptome data of BrainSpan was performed using the filtered gene list (average reads per kilobase per million mapped reads (RPKM) $>0.05$ over all developmental stages) as background.

\section{Enrichment analysis}

Enrichment scores for GO terms were calculated as followed: $(a / b) /[(c-a) /(d-b)]$, where $a$ is the number of genes in the ataxia category associated with that GO term, $b$ is the total number of genes in the ataxia category, $c$ is the total number of genes in the genome associated with that GO term or, for GO analysis of BrainSpan data, the total number of genes associated with that GO term remaining after filtering out low expressed genes from BrainSpan data and $d$ is the total number of annotated human genes present in Ensembl (20313 genes) or, for GO analysis of BrainSpan data, the total number of genes remaining after filtering out low expressed genes from BrainSpan data (16956 genes).

\section{Protein-protein interaction network}

Protein-protein interactions (PPI) between CA genes were obtained from GeneMANIA, HPRD and BioGrid and included physical interactions, predicted interactions, shared protein domains and pathways. ${ }^{8-10}$ All interactions were combined and assembled in a reference network using Cytoscape ${ }^{11}$ (V.3.1.1.) and duplicates were removed from the reference network.

\section{Physical interaction enrichment score}

We used the physical interaction enrichment (PIE) algorithm to account for biases in the number of reported protein interactions for disease-associated genes in the generated reference PPI network. ${ }^{12}$ PIE scores and associated $\mathrm{p}$ values were calculated against 10000 random protein groups obtained by numbermatched subsamplings selected from the reference PPI network for all four ataxia gene categories, as previously described. ${ }^{13} 14$

\section{BrainSpan developmental transcriptome analysis}

The publicly available developmental transcriptome RNA sequencing (RNAseq) data from the Human BrainSpan atlas was used for ataxia gene expression analysis. BrainSpan provides RNAseq count data represented as RPKM of 11 targeted neocortical human brain regions and 5 targeted non-neocortical brain regions. Details on samples, sequencing protocols and RNA expression analysis can be found at the BrainSpan website (http://www.brainspan.org). First, low expressed genes, with an average expression $<0.05$ RPKM over brain developmental stages and regions, were filtered out. Data were then binned into nine stages, spanning important developmental milestones of the prenatal and postnatal human brain. On average, three samples per developmental stage and brain structure were available (online supplementary table S2). Samples were not sex-matched. EdgeR $^{15}$ (V.3.16.5) and Limma ${ }^{16}$ (V.3.30.7), provided by the online service Bioconductor, were used to identify the differentially expressed genes between the 16 brain regions separately over the developmental stages. Genes were considered to be differentially expressed between two brain regions if their adjusted $\mathrm{p}$ value passed the $<0.05$ threshold (Benjamini-Hochberg correction for the number of differentially expressed genes). No Log2FC threshold was set for the differential expression analysis. Graphpad Prism V.5.0 contingency tables and Chi Square and Fisher's exact (in case of groups containing less than five genes) two-tailed tests were used to calculate whether ataxia gene expression was significantly enriched in the cerebellum compared with the rest of the brain. GENE-E (V.3.0.215, www.broadinstitute.org) was used to hierarchically cluster average RPKM values of ataxia genes in the cerebellum over the nine defined developmental stages.

\section{RESULTS}

\section{A systematic catalogue of genes associated with progressive cerebellar ataxia}

We generated a manually curated systematic catalogue of 103 disorders consistently associated with progressive cerebellar ataxia, corresponding to 71 annotated protein-coding genes (online supplementary table S1). Of the 71 genes that met our criteria, mutations in 42 of the genes follow recessive inheritance and mutations in 31 of the genes follow dominant inheritance. Two genes, SPTBN2 and AFG3L2, have been described in both dominant and recessive ataxia. Generally, mutations associated with recessive ataxia are loss of function mutations, whereas dominant ataxias can be caused by a combination of gain and/ or loss of function mutations. Several genetic ataxias are caused by unstable repeat expansions that can occur in non-coding and coding regions of the genome. Eight of these (ATXN1, ATXN2, ATXN3, ATXN7, ATXN8, CACNA1A, TBP and ATN1) contain/ represent translated trinucleotide repeats that encode for polyQ residues and follow dominant inheritance.

\section{Genes involved in ataxia function in common biological processes}

Differences in the type of mutations and phenotypes between recessive and dominant ataxias suggest that in spite of shared clinical features, different biological mechanisms might underlie these disorders. ${ }^{25}$ To examine whether recessive, dominant and polyQ ataxia genes are associated with distinct biological functions, GO analysis was performed on the three gene categories. Recessive ataxia genes were significantly enriched for catalytic activity, acting on DNA $\left(\mathrm{p}=2.23 \mathrm{e}^{-4}\right)$, DNA repair $\left(\mathrm{p}=2.0 \mathrm{e}^{-4}\right)$ and cellular response to stress $\left(\mathrm{p}=8.3 \mathrm{e}^{-4}\right)$ (figure 1A). Dominant ataxia genes were significantly enriched for nuclear matrix $(p=0.031)$, somatodendritic compartment $(p=0.015)$ and dendrite $(p=0.018)$ (figure 1B). Ataxia genes encoding poly $Q$ expansions were significantly enriched for regulation of cellular biosynthetic process $(p=0.025)$, nuclear periphery $\left(p=5.56 \mathrm{e}^{-5}\right)$, nuclear matrix $\left(\mathrm{p}=2.31 \mathrm{e}^{-5}\right)$ and nuclear inclusion body $(p=0.007)$ (figure 1C). Despite these differences, the shared clinical hallmarks of genetic ataxias suggest that ataxia genes/ proteins might affect common biological pathways or processes. To identify such common biological themes, we also analysed the combined ataxia gene catalogue. We found a significant enrichment for most of the GO terms revealed by the separate analyses of genes underlying ataxia subtypes, including cellular response to stress $(p=0.002)$ and DNA repair $\left(p=6.52 \mathrm{e}^{-4}\right)$ (recessive), somatodendritic compartment $(\mathrm{p}=0.004) \quad$ (dominant) and nuclear inclusion body $(\mathrm{p}=0.034)$ (polyQ) (figure 1D). Interestingly, analysis of the contribution of recessive and dominantly inherited ataxia genes to the identified GO terms revealed a shared contribution to nearly all processes (figure 1D), 
Recessive Cerebellar Ataxias

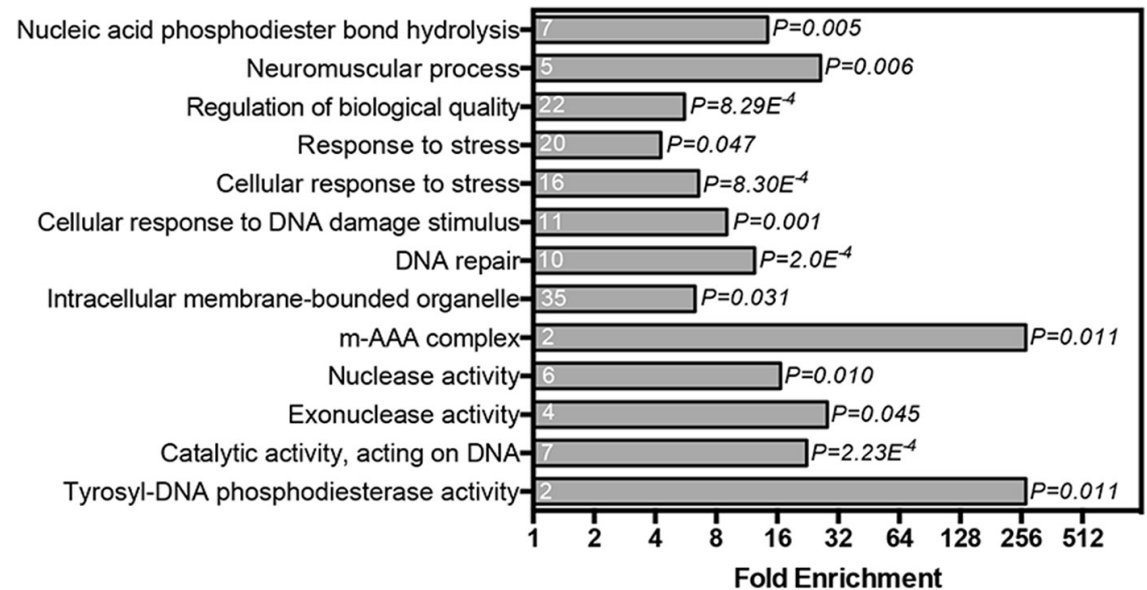

B

\section{Autosomal Dominant Cerebellar Ataxias}

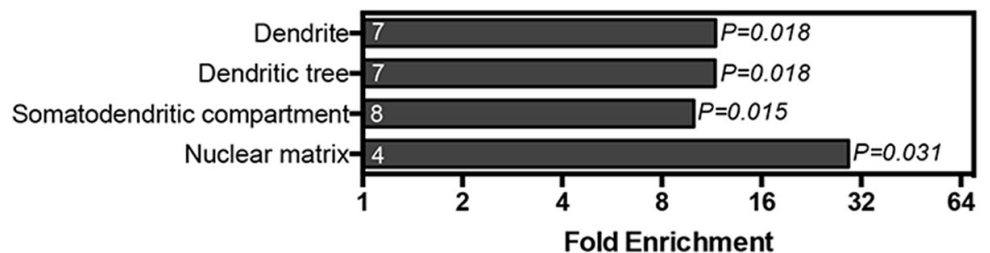

C

Autosomal Dominant Cerebellar Ataxias with PolyQ expansion
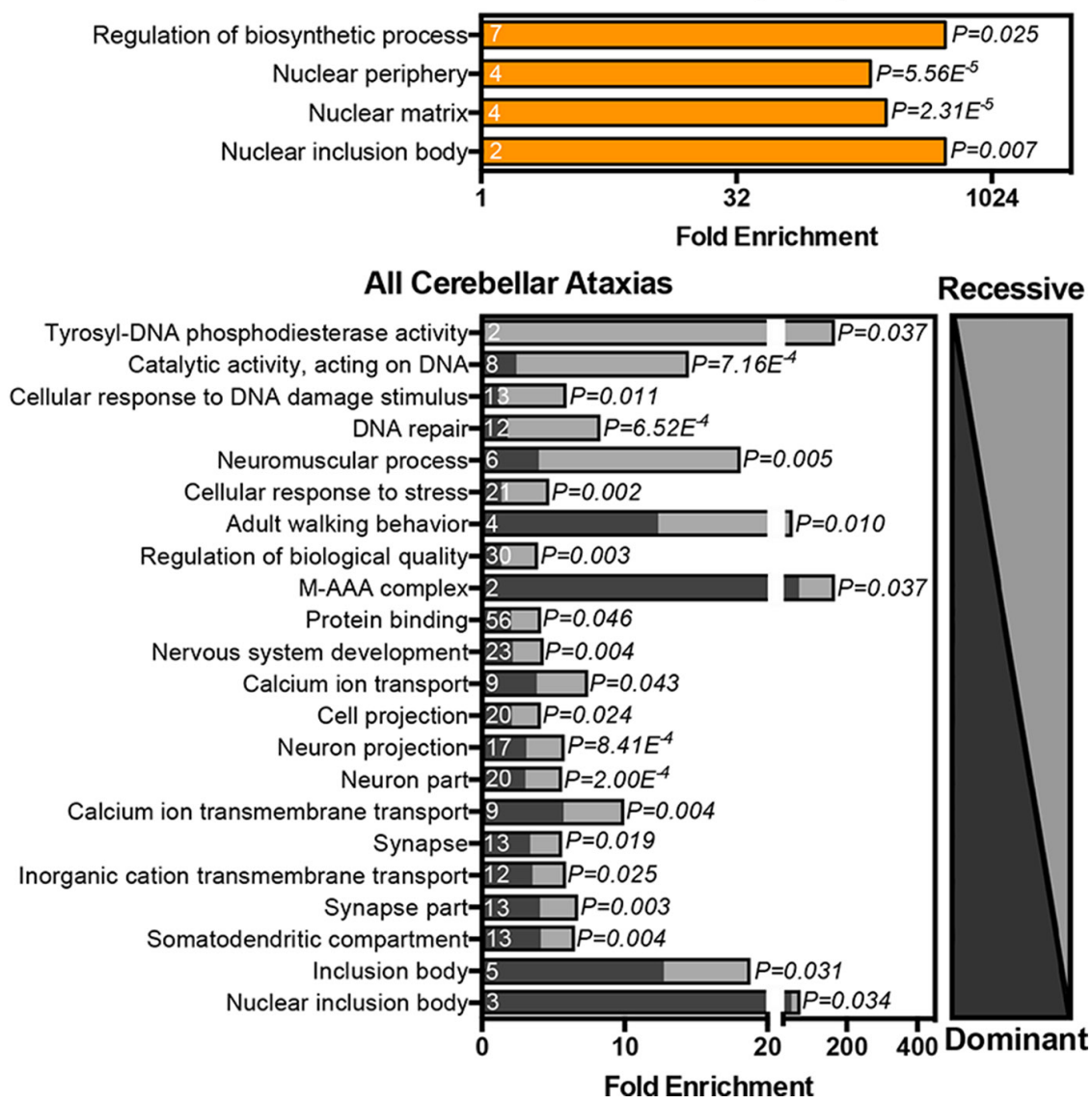

Figure 1 Cerebellar ataxia genes function in common biological processes. Gene ontology (GO) terms significantly enriched among (A) recessive ataxia genes, (B) dominant ataxia genes, (C) dominant ataxia genes with polyglutamine (polyQ) expansion, (D) all ataxia genes (all GO terms passed Bonferroni correction for multiple testing, $\mathrm{p}<0.05$ ). 
A

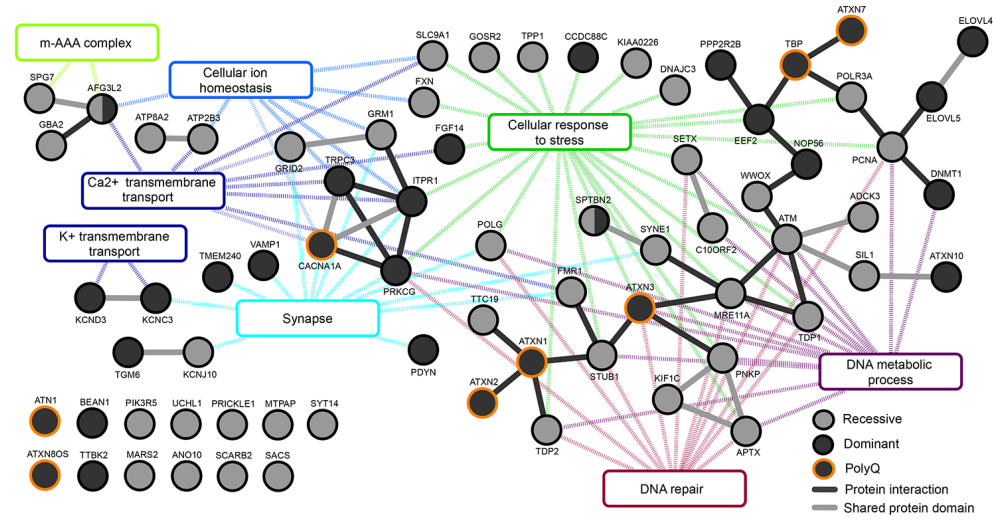

B

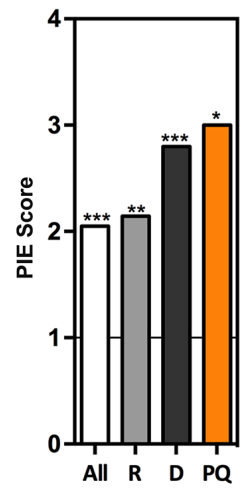

Figure 2 Cerebellar ataxia proteins show high connectivity on protein level and function in common processes. (A) Interaction network of ataxia proteins (black solid lines: direct protein interaction, grey solid lines: proteins with similar domains, dotted lines: interaction of protein with gene ontology (GO) term). (B) Physical interaction enrichment (PIE) score of all, recessive (R), dominant (D) and polyglutamine (PQ) ataxia proteins. ${ }^{*} \mathrm{P}<0.05,{ }^{* *} \mathrm{p}<0.01,{ }^{* * *} \mathrm{p}<0.001$, based on 10000 random repetitions.

supporting an overlapping molecular pathology underlying both dominant and recessive ataxias. Moreover, several GO terms, such as those linked to calcium ion transmembrane transport $(\mathrm{p}=0.004)$, neuron projection $\left(\mathrm{p}=8.41 \mathrm{e}^{-4}\right)$ and adult walking behaviour $(p=0.010)$, were only highlighted if the analysis was applied to the complete catalogue of ataxia genes and might be more representative of the shared hallmarks between recessive and dominant progressive cerebellar ataxias. Alternatively, they were not detected in the analyses of the separate groups due to limited statistical power of the smaller group sizes.

\section{Genes involved in ataxia show high connectivity on the protein level}

Physical and functional interactions of proteins provide the basis of biological pathways and are crucial to understand cellular function. We evaluated whether our catalogue of ataxia proteins shows significant molecular connectivity. For this, we collected PPI data from three large databases and combined these into a reference network (figure 2A). We found that 46 of the proteins physically interacted with other proteins present in the ataxia catalogue. Of these 46 proteins, 17 proteins were connected in small modules (pairs, trimers and hexamers), whereas 29 proteins formed a single major network with 30 interactions (figure 2A). Interestingly, within these modules and networks, proteins associated with recessive, dominant and polyQ progressive ataxia are jointly represented, demonstrating a biological overlap regardless of inheritance type or mutational mechanism (figure 2A). To assess the significance of the identified connectivity, PIE scores and associated $\mathrm{p}$ values were calculated for all, and separately for recessive, dominant and polyQ ataxia proteins. This analysis revealed that ataxia proteins as a whole group interact twofold more than randomly expected $(\mathrm{p}<0.001)$ (figure $2 \mathrm{~B})$. Recessive ataxia proteins interacted 2.1 -fold more $(\mathrm{p}<0.001)$, dominant ataxia proteins interacted 2.8 -fold more $(\mathrm{p}<0.001)$ and polyQ-associated ataxia proteins interacted 3 -fold more $(\mathrm{p}<0.05)$ (figure $2 \mathrm{~B})$. Thus, ataxia proteins are significantly interconnected. Since interactions between proteins underlie biological processes and pathways, we continued examining whether these represent specific biological processes. Individual small protein modules within the ataxia interactome (figure 2A, left side) were significantly enriched for processes such as calcium ion homeostasis $(p=0.003)$ and calcium ion transmembrane transport activity $(\mathrm{p}=0.006), \mathrm{m}$-AAA complex $\left(\mathrm{p}=4.36 \mathrm{e}^{-6}\right)$, unfolded protein binding $(\mathrm{p}=0.024)$, potassium ion transport $(\mathrm{p}=0.026)$ and potassium channel activity $(\mathrm{p}=0.007)$ (figure $2 \mathrm{~A}$, left side). The large module within the ataxia interactome was significantly enriched for DNA repair $\left(\mathrm{p}=1.21 \mathrm{e}^{-5}\right)$, DNA metabolic process $\left(p=1.78 \mathrm{e}^{-4}\right)$, cellular response to stress $(p=0.009)$ and nuclear inclusion body $(\mathrm{p}=0.001)$ (figure $2 \mathrm{~A}$, right side). Together, these results illustrate that ataxia proteins function in common biological networks and processes.

\section{A subset of ataxia genes shows high relative expression specifically in the cerebellum}

The basis for the preferential regional vulnerability of neurons in the cerebellum in genetic ataxias is mostly unknown. Temporal and spatial patterns of ataxia gene expression in the brain may significantly contribute to specific or preferential cerebellar degeneration when disturbed. To address this, we turned to the publicly available BrainSpan Transcriptional Atlas of the Developing Human Brain, as resource. After exclusion of low expressed genes, a transcription matrix of 16956 genes representing the 16 brain regions was left that was binned into nine different developmental stages (figure 3A). We then calculated for each of the 16956 genes per developmental period whether it was differentially expressed in the cerebellum, compared pairwise with any of the other 15 brain regions. From the resulting matrix, the ataxia genes were extracted and the percentage of ataxia genes differentially expressed (adj. $p<0.05)$ in the cerebellum compared with one or more other brain regions was calculated (data summary: online supplementary table S3, data per stage: online supplementary tables S4-S12) and visualised in a heatmap (figure $3 \mathrm{~B}$ ). Of note, the expression levels of three ataxia genes, TGM6, PIK3R5 and MTPAP, did not pass the threshold of very low expressed genes and were therefore excluded from further analyses. The expression of most ataxia genes in the cerebellum was not different from any of the other 15 brain regions (online supplementary table S3), and their average did not differ from the average genome-wide expression (online supplementary figure S1). However, the vast majority of those ataxia genes that did show differential expression to other brain regions, did so to all 15 of them (figure 3B, black square, online supplementary table S3). Their high relative expression level was specific to the cerebellum, suggesting their distinct requirement in this region of the brain. Interestingly, this pattern showed a sharp onset at birth, suggesting that these genes serve cerebellar function specifically at postnatal stages (figure $3 \mathrm{~B}$, black square). 
A

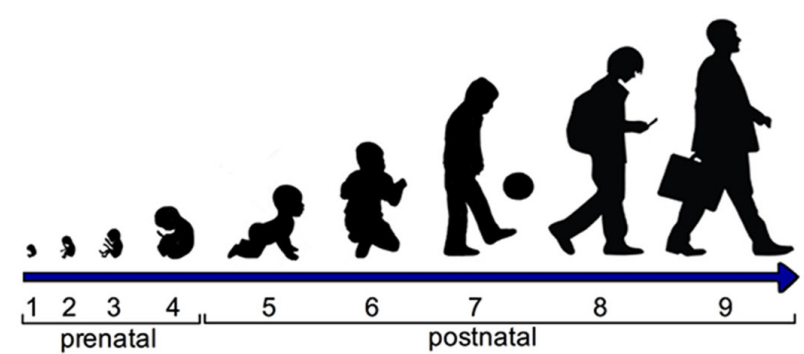

B

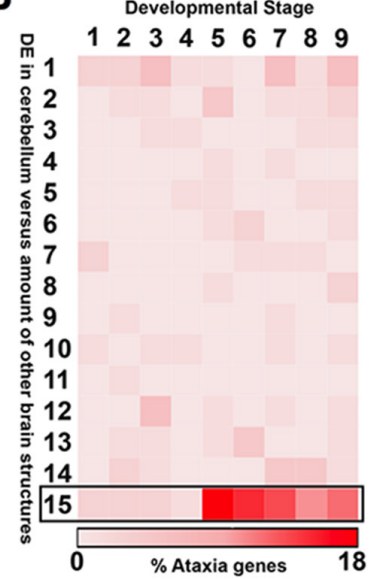

C

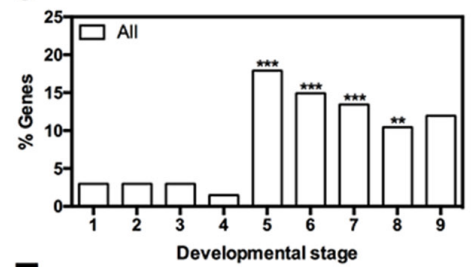

E

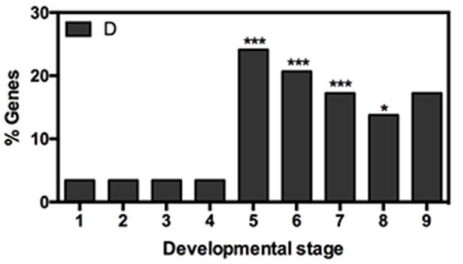

Developmental stage:

1: Early prenatal $\quad 12-13 \mathrm{pcw}$

2: Early midprenatal $16-17 \mathrm{pcw}$

3: Late midprenatal $19-24 \mathrm{pcw}$

4: Late prenatal $35-37 \mathrm{pcw}$

5: Infancy $\quad 0-1$ years

6: Early childhood $\quad 2-4$ years

7: Late childhood $\quad 8-11$ years

8: Adolescence $13-19$ years

9: Adulthood $21-40$ years

D

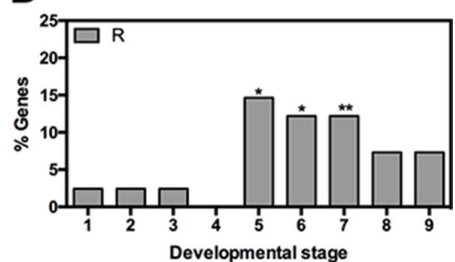

$\mathbf{F}$

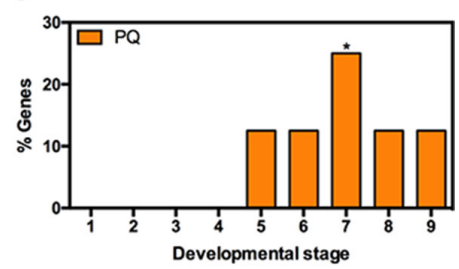

G

GO-terms linked to CA genes that show enriched expression in the cerebellum
GO-terms linked to CA genes that do not show enriched expression in the cerebellum

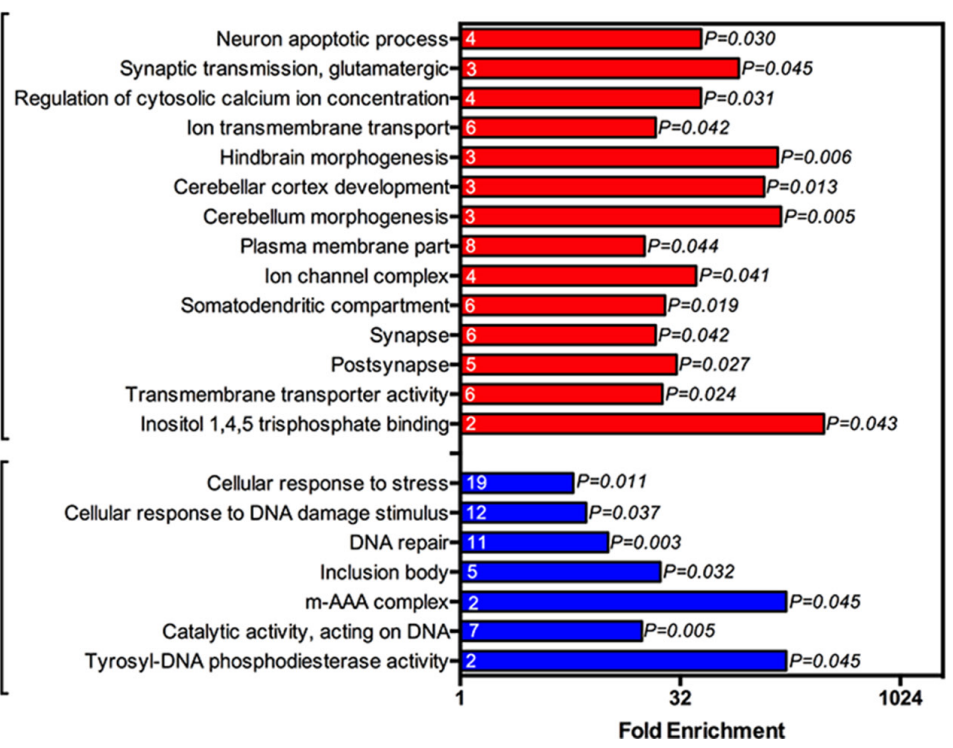

Figure 3 Cerebellar ataxia gene expression is enriched in the postnatal cerebellum. (A) Nine developmental stages were used for analysis of developmental human BrainSpan expression data. (B) Heatmap displaying \% of ataxia genes differentially expressed in the cerebellum compared with indicated amount (Y-axis) of other non-cerebellar brain regions (a single up to 15) for developmental stages 1-9. (C-F) Percent of ataxia genes that show significant enriched expression in the cerebellum compared with 15 other brain regions for described developmental stages ((C) all ataxia genes, (D) recessive ataxia genes, (E) dominant ataxia genes, (F) dominant ataxia genes with polyglutamine (PQ) expansion, ${ }^{*} p<0.05,{ }^{* *} p<0.01,{ }^{* * *} p<0.001$. (G) Significantly enriched gene ontology (GO) terms for ataxia genes elevated in the cerebellum (red) and ataxia genes not elevated in the cerebellum (blue).

Elevated cerebellar-specific expression predisposes to ataxia We continued determining whether elevated relative expression in the cerebellum predisposes to ataxia. To address this, we calculated whether the amount of ataxia genes that were specifically elevated in the cerebellum for each developmental stage was higher than randomly expected, considering the genomewide frequency of cerebellar elevated genes. Ataxia genes were more frequently elevated in the cerebellum during the postnatal stages 5-9 (infancy: $p<0.001$, early childhood: $p<0.001$, late childhood: $p<0.001$ and adolescence: $p<0.01)$ than randomly expected (figure 3C). The following genes were specifically elevated in the cerebellum during one or more of the analysed developmental stages: ADCK3, ATM, ATN1, CACNA1A, DNMT1, GRID2, GRM1, ITPR1, KCNC3, KCND3, SPTBN2, SYNE1 and TRPC3 (online supplementary tables S3-S12). To further examine whether a specific mode of inheritance or mutational mechanism was underlying this group of genes, the analysis was repeated for the recessive, dominant and polyQ ataxia gene categories. Also here, the expression of genes involved in either of these three categories was enriched in the cerebellum 
during postnatal development stages (figure 3D-F), suggesting that all of them contribute to the finding of cerebellum-specific postnatal enrichment of ataxia gene expression. Finally, we asked whether the genes that were not specifically elevated in the cerebellum during one of the nine developmental stages, were specifically elevated in one of the other 15 brain regions. We found that a small group of ataxia genes was specifically elevated in either the thalamus (GBA2, UCHL1, PRICKLE1 and $A N O 10)$ or striatum (CCDC88C and PDYN) during certain developmental stages compared with rest of brain. Not a single ataxia gene was significantly elevated in one of the remaining 13 specific brain regions. In summary, systematic analyses of brain expression across brain regions revealed that the expression of a subgroup of ataxia genes is specifically enriched in the postnatal cerebellum, likely driving the pathological features of these disorders.

\section{Cerebellar-specific expression patterns separate ataxia genes in distinct biological modules}

We next asked which functional biological modules are underlying the identified group of genes with cerebellar-specific expression. GO term analysis was performed on the lists of ataxia genes that showed enriched cerebellar expression during one or more of the developmental stages and ataxia genes that did not (online supplementary tables S3-S12). This revealed that ataxia genes that were specifically elevated in the cerebellum during one of the developmental stages were significantly enriched for neuronal and ion-related processes such as neuron apoptotic process $(p=0.030)$, hindbrain morphogenesis $(p=0.006)$, regulation of cytosolic calcium ion concentration $(p=0.031)$, synapse $(p=0.042)$ and transmembrane transporter activity $(p=0.024)$ (figure $3 G$ ). Ataxia genes that were not specifically elevated in the cerebellum during one of the developmental stages were significantly enriched for processes such as DNA repair $(p=0.003)$, cellular response to stress $(p=0.011)$ and inclusion body $(p=0.032)$ (figure $3 G)$.

\section{Temporal gene expression patterns in the cerebellum cluster genes involved in progressive ataxia into two groups}

Finally, we also explored developmental expression profiles of ataxia genes in the cerebellum over time, to examine whether this provides further clues about dependence of the cerebellum on certain biological processes during specific stages of development (figure 4A, B). We hierarchically clustered the temporal expression patterns of ataxia genes in the cerebellum over the nine developmental stages, which unbiasedly separated the genes in two distinct clusters (figure 4B). Genes present in cluster 1 showed significant higher expression during prenatal stages compared with genes in cluster 2 , and genes present in cluster 2 showed significant higher expression during postnatal stages compared with genes present in cluster 1 (figure 4A). Recessive, dominant and polyQ-associated ataxia genes contributed randomly to the two clusters (data not shown). However, genes expressed higher during prenatal stages (cluster 1) were enriched for DNA repair $(\mathrm{p}=0.031)$ and cellular response to stress $(p=0.035)$, whereas genes with higher postnatal expression (cluster 2) were enriched for processes related to the cellular component synapse $(\mathrm{p}=0.029)$, calcium ion transmembrane transport $(\mathrm{p}=0.035)$ and metal ion transport $(\mathrm{p}=0.028)$ (figure 4C). Ataxia genes can thus be distinguished based on their temporal expression profiles in the cerebellum, which links to specific biological processes.

\section{DISCUSSION}

We have here systematically mapped shared molecular pathways, processes and expression characteristics among ataxia causing genes, to increase our understanding of the biology of genetic ataxias and to identify mechanistic hubs that can serve as targets for therapeutic interventions. In comparison to previous studies, ${ }^{2-5}$ we generated a manually curated catalogue of genes involved in genetic ataxia and performed analyses across dominant and recessive forms of the disorder. Furthermore, we applied a strategy that integrated ataxia gene expression in developing human brain, GO and protein interaction network analysis, to get a comprehensive understanding of the vulnerability of the cerebellum and the molecular modules and processes affected in genetic ataxias.

Data in this study highlight the different biological processes that are implicated in recessive, dominant and polyQ ataxias, with recessive ataxias linked to cellular response to stress and DNA repair-related processes; dominant ataxias to dendrite-related processes and polyQ ataxias to nuclear inclusion body. The deleterious effect of the type of mutation on the protein, the expression timing profile, specificity and levels of the affected protein in the cerebellum and the sensitivity of the cerebellum to disruption of these processes might explain these different findings. Despite this, we found a shared contribution of recessive and dominant ataxia genes to nearly all biological processes, while processes such as calcium ion transmembrane transport were only enriched when applying GO analysis to the complete ataxia gene panel. In agreement with previous studies, ${ }^{34}$ we found that ataxia genes operate in networks with significantly enriched protein connectivity, demonstrating global coherence independent of inheritance mode or mutational mechanism. Notably, polyQ proteins interacted directly with other non-polyQ dominant and recessive ataxia proteins. This indicates that, in addition to common toxic gain-of-function mechanisms ${ }^{17}$ such as the formation of nuclear inclusion bodies, the disruption of the biological processes that these genes operate in likely contributes to the disease pathogenesis. This notion derived from our systematic analysis is supported by gene-focused studies, for example, of mice models of SCA1, where ATXN1 loss-of-function phenotypes were very similar to ATXN1 gain-of-function phenotypes, and of SCA17 models demonstrating that impaired transcriptional activity of polyQ-expanded TBP contributes to disease pathogenesis. ${ }^{18-20}$ Together, our findings strongly support an overlapping molecular pathology between recessive and dominant ataxia subtypes. The identified protein modules represent different biological processes and ataxia proteins can broadly be separated in two themes: a large stress module and smaller ion homeostasis/ synapse modules. The common end point of these modules is progressive degeneration of the cerebellum, and analysis of ataxia gene expression in the developing human brain suggested that these two modules might contribute differently to cerebellar vulnerability, depending on their specific and temporal cerebellar expression pattern (figure 5). Brain expression analysis demonstrated that elevated gene expression specifically in the postnatal cerebellum predisposes to ataxia. Genes with this pattern of expression are significantly overrepresented among ataxia genes. The normally high gene activity levels may explain the specific vulnerability of the cerebellum to deleterious mutations in these genes. Interestingly, the corresponding genes encode ion channels, calcium receptors, calcium-activated proteins enriched for transmembrane transporter activity and regulators of cytosolic calcium ion concentration. Most of them 
A

B
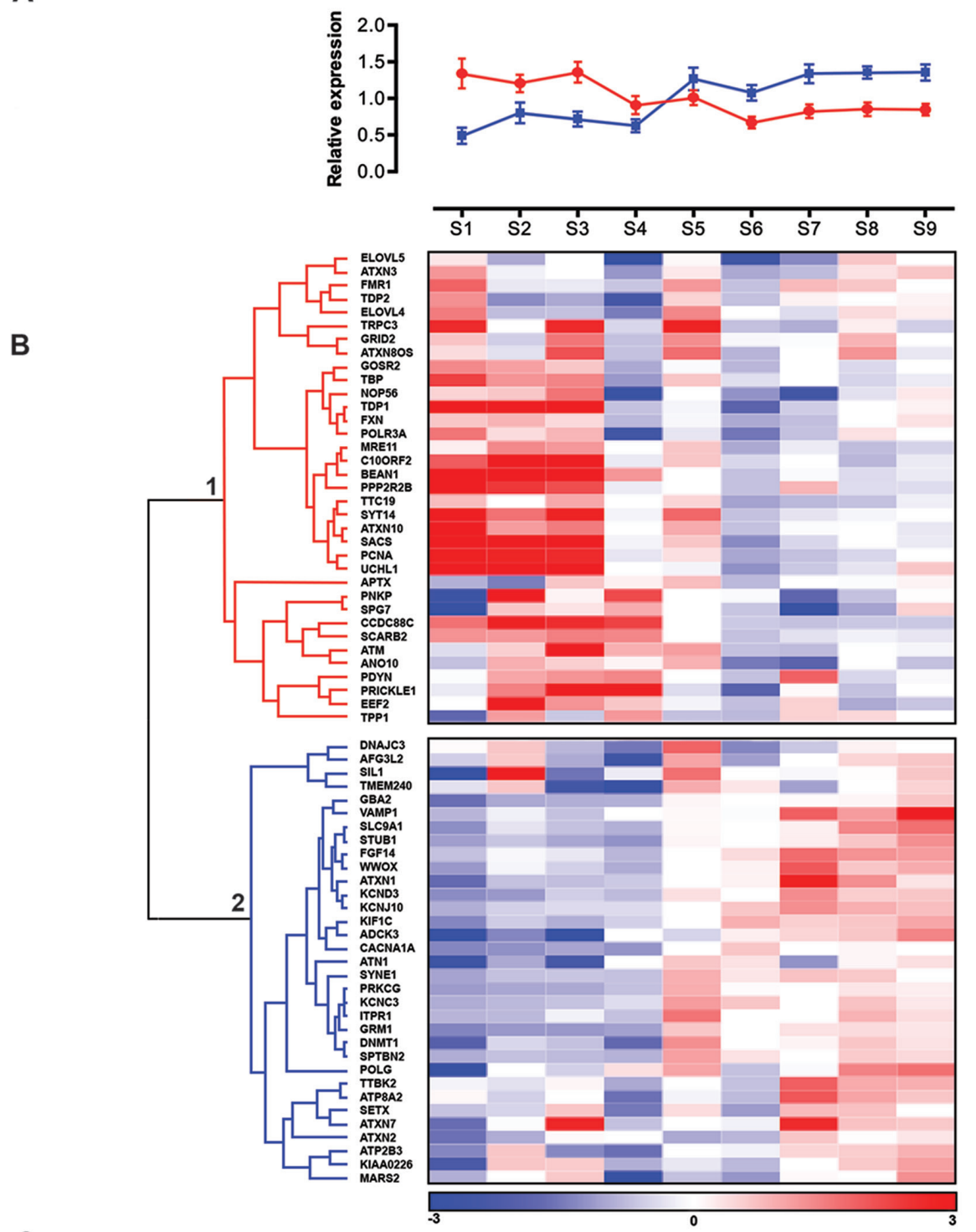

C

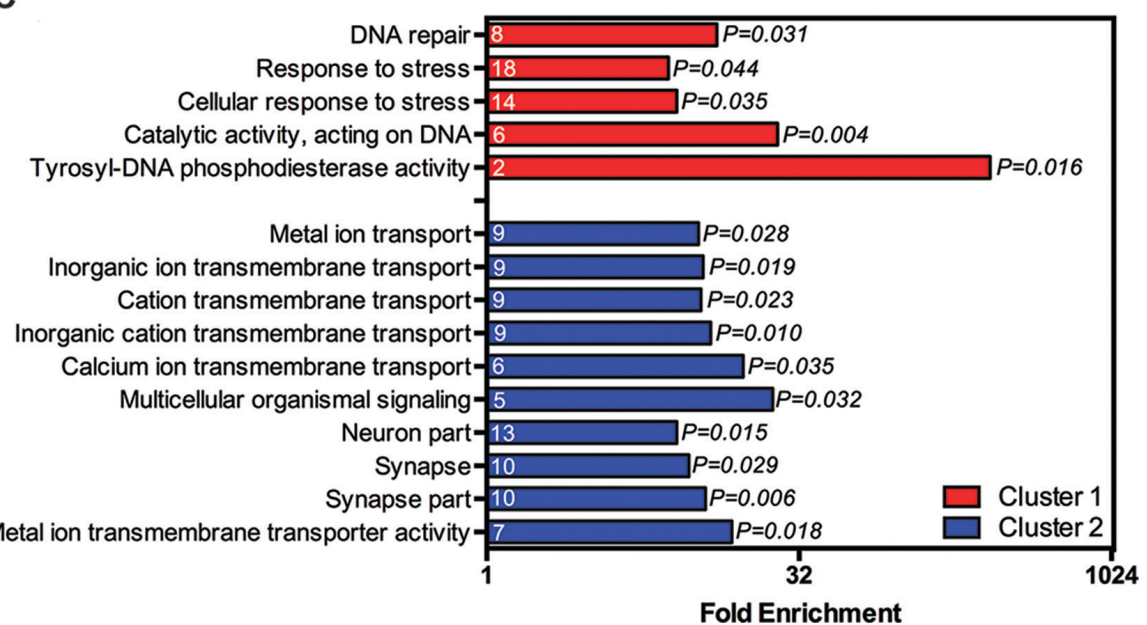

Figure 4 Ataxia genes can be separated in two distinct clusters based on their temporal expression levels in the cerebellum. (A) Average reads per kilobase per million mapped reads (RPKM) value for ataxia genes present in cluster 1 and cluster 2 of figure 4B, for the indicated developmental stages. (red: cluster 1, blue: cluster 2, error bars represent SD). (B) Hierarchical clustering of ataxia gene expression levels during cerebellar development using Spearman's correlation. Data were obtained from BrainSpan and mean RPKM values were calculated for the indicated developmental stages. Heatmap colour codes are based on median RPKM value per row (developmental stage), divided by the row standard deviation (blue: low expression in cerebellum compared with median, red: high expression in cerebellum compared with median). (C) Significantly enriched gene ontology (GO) terms for cluster 1 and cluster 2 from figure 4B (all GO terms passed Bonferroni correction for multiple testing, $\mathrm{p}<0.05$ ). 


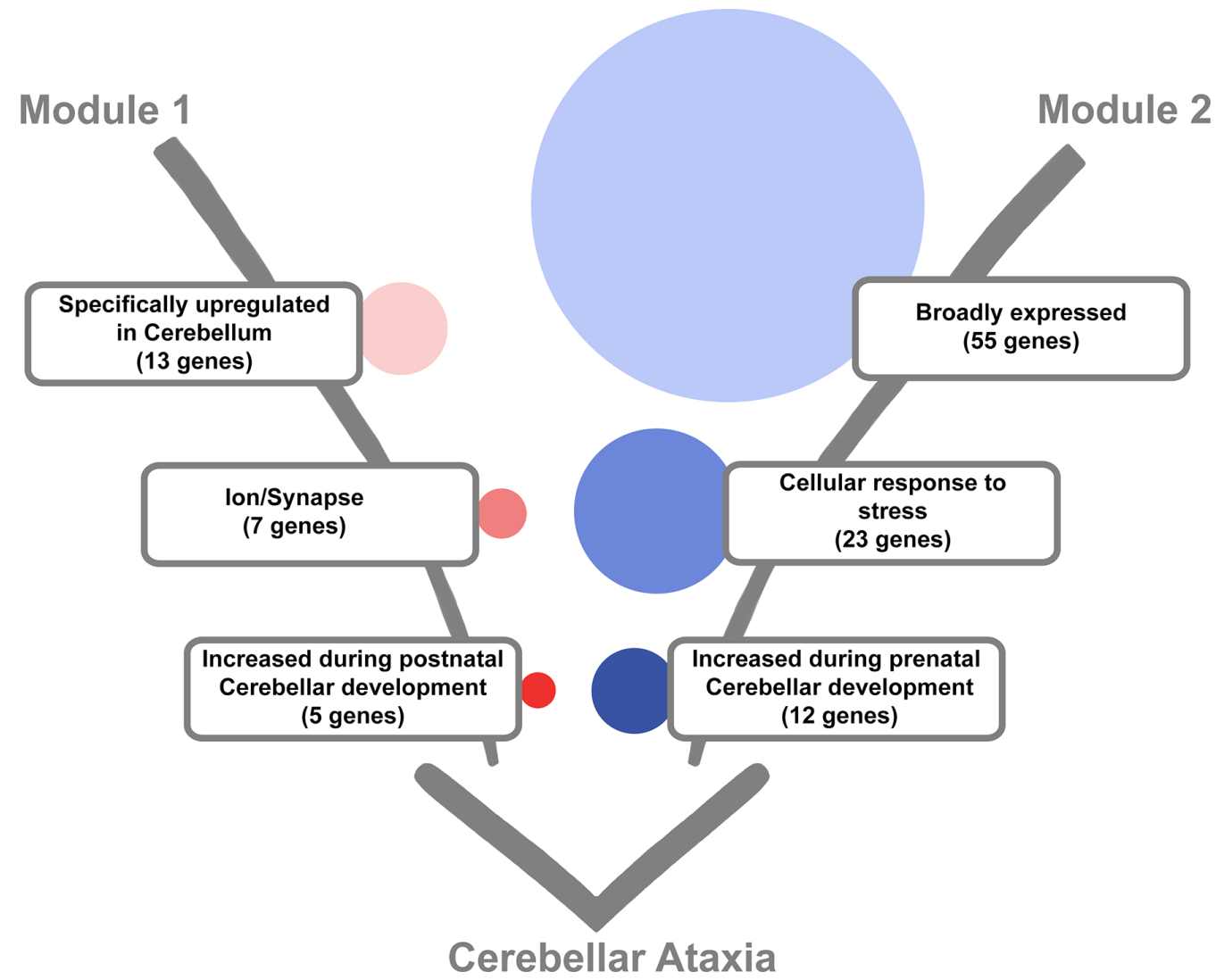

Figure 5 Ataxia genes can broadly be divided in two themes that affect neuronal homeostasis and when disrupted predispose to progressive cerebellar ataxia. Module 1: 13 genes are specifically elevated in the cerebellum during one or more developmental stages. Of these 13 genes, 7 are linked to lon/ Synapse function and 5 out of these 7 genes showed increased expression during postnatal cerebellar development compared with prenatal cerebellar development. Module 2: 55 genes do not show increased expression in the cerebellum. Of these 55 genes, 23 genes are linked to cellular response to stress and 12 out of these 23 genes show increased expression during prenatal cerebellar development compared with postnatal cerebellar development (data displayed in figures 2-4).

are part of the smaller ion homeostasis/synapse module we identified (figure 3). These results are similar to those obtained by Bettencourt et al, demonstrating that disruptive calcium signalling is important in a dominant ataxia gene, transcript-enriched, co-expression module relevant to Purkinje cells (PC). ${ }^{4}$ The cerebellum contains a unique neuronal cell type, the $\mathrm{PC}$, that is central to ataxia pathobiology. PCs might be particularly vulnerable for alterations in ion homeostasis due to their extensive dendritic arbour that exhibit intense and highly regulated firing properties. $^{21-23}$ The striking complexity of PC firing regulates sensorimotor integration and is highly dependent on calcium channels and calcium-activated potassium channels. ${ }^{21}$ In several ataxia models, PCs show reduced firing rate and dysregulated calcium homeostasis, even before the onset of clinical symptoms and PC degeneration. ${ }^{17} 2425$ Reduced PC excitation enhances the excitability of efferent deep cerebellar nuclei neurons and this is sufficient to cause cerebellar ataxia even in the absence of PC degeneration. ${ }^{26}$ This shows that calcium homeostasis in PCs is crucial for proper sensorimotor integration and disruption of these processes likely affects PC firing properties, eventually leading to cerebellar degeneration and the onset of ataxia. Calcium is also an important messenger in intracellular signalling pathways, proteostasis mechanisms at ER and Golgi membranes and mitochondrial activity. ${ }^{27} 28$ The metabolic activity of PCs is high. ${ }^{29}$ Deficits in neuronal energy production and intracellular organelle systems that influence ion fluxes may thus be further factors that account for increased vulnerability of PCs. The energy provided for metabolism is provided by mitochondrial oxidative phosphorylation in the form of ATP. Increased calcium uptake by mitochondria leads to extensive oxidative phosphorylation and overproduction of reactive oxygen species (ROS). ${ }^{27}{ }^{28}$ ROS overproduction in turn can lead to detrimental oxidative modifications of lipids, proteins and nucleic acids. ${ }^{30} 31$ Interestingly, the large identified protein module is enriched for the cellular stress response, and proteins present in this module are among others involved in mitochondrial maintenance, DNA repair, unfolded protein response and regulation of apoptotic and autophagic processes. We found that overall, these genes were not more elevated in the cerebellum during any of the analysed developmental stages. Thus, elevated cerebellar gene expression cannot account for preferential cerebellar degeneration. This suggests that, compared with other neuronal cell types, the PCs might be more vulnerable to insults related to a distorted cellular stress response. Interestingly, approximately half of the cellular stress response genes show higher expression during embryonic stages, arguing for a developmental predisposition in these cases. Ataxia genes with a function in DNA repair were abundantly represented in the stress module (figure 3). DNA breaks arising from oxidative damage are a major threat for the genome stability of mature postmitotic neurons. They are usually repaired by base excision repair and single strand break repair (SSBR). ${ }^{32}$ Interestingly, genetic ataxias such as SCAN1 and AOA1, are associated with DNA SSBR deficiencies, and animal models of these show 
increased sensitivity to ROS. ${ }^{29} 32$ The genes mutated in these disorders, TDP1 and APTX, do not show enriched cerebellar expression during any of the nine analysed developmental stages, which may indicate that SSBR is a key homeostatic process required in PCs for reasons unrelated to expression, such as their high metabolic activity, oxidative load and intrinsic firing properties. There is also a distinct subset of genes implicated in recessive ataxias that are involved in double strand break (DSB) repair. Double-stranded DNA breaks commonly occur during rapid proliferation of central nervous system (CNS) development and are less likely to occur in the matured nervous system. ${ }^{33}$ The cerebellum might be in particular vulnerable to disruption of DSB repair due to its development up until the first postnatal years, which will lead to the formation of the most abundant cell type in the CNS: the cerebellar granule neurons. ${ }^{29}$ This postnatal period of rapid and massive cell proliferation may generate replication stress-associated DNA damage that might affect the cerebellar granule neurons, and indirectly other cerebellar cell types such as the PCs to which they signal. ${ }^{29}$ Interestingly, ATM, a kinase that is involved in detection of DSBs and is mutated in ataxia telangiectasia (AT), shows enriched expression in the cerebellum exactly during this postnatal developmental period. Since ATM is involved in DSB-induced apoptotic signalling, dysfunctional neurons may fail to be efficiently eliminated in the early AT cerebellum, and degenerate only later on. ${ }^{32} 3435$ This is in agreement with the early neurological problems and loss of the cerebellar granule neurons, the molecular cell layer and PCs in AT. ${ }^{32} 3637$ Nonetheless, since ROS-induced DNA damage can also include DSBs and antioxidant treatment can promote the survival of cultured ATM-deficient PCs, DSB repair might also be required for cerebellar maintenance. ${ }^{32} 38$ Therefore, DSB repair is likely a key homeostatic process that maintains PC integrity.

In conclusion, while a number of molecular processes are involved in progressive cerebellar ataxia pathology, these intersect and form a common end point: disrupted neuronal homeostasis, to which the cerebellum is either exclusively susceptible, or more than other brain regions. More experimental data are required to understand the dependence of the cerebellum on different aspects of neuronal homeostasis, such as calcium signalling, ROS and DNA repair, particularly in absence of region-specific expression levels. However, based on the here identified biological themes, it seems conceivable to propose therapeutic interventions that target deregulated calcium and ROS levels, or mechanisms that can decrease the harmful downstream effects of these deleterious insults.

\section{Acknowledgements The authors would like to thank P Cizek for the PIE score script and C Gilissen for advice on the expression analysis.}

Contributors All authors conceived the study. IE designed the analyses, collected, analysed and interpreted the data and drafted the manuscript. BPvdW contributed to the design of the gene list. BPvdW and AS interpreted the results, and critically reviewed the manuscript. All authors read and approved the final version of the manuscript.

Funding This research was supported by the E-RARE-3 Joint Transnational Call grant 'Preparing therapies for autosomal recessive ataxias' (PREPARE; ZonMW 9003037604 to BPvdW and AS) and by a Donders (enter for medical Neuroscience/ Radboud University Medical Center junior researcher grant.

Competing interests None declared.

Patient consent for publication Not required.

Provenance and peer review Not commissioned; externally peer reviewed.

Open access This is an open access article distributed in accordance with the Creative Commons Attribution Non Commercial (CC BY-NC 4.0) license, which permits others to distribute, remix, adapt, build upon this work non-commercially, and license their derivative works on different terms, provided the original work is properly cited, appropriate credit is given, any changes made indicated, and the use is non-commercial. See: http://creativecommons.org/licenses/by-nc/4.0/.

\section{REFERENCES}

1 Jayadev S, Bird TD. Hereditary ataxias: overview. Genet Med 2013;15:673-83.

2 Didonna A, Opal P. Advances in sequencing technologies for understanding hereditary ataxias: a review. JAMA Neurol 2016;73:1485-90.

3 Lim J, Hao T, Shaw C, Patel AJ, Szabó G, Rual JF, Fisk CJ, Li N, Smolyar A, Hill DE, Barabási AL, Vidal M, Zoghbi HY. A protein-protein interaction network for human inherited ataxias and disorders of Purkinje cell degeneration. Cell 2006;125:801-14.

4 Bettencourt C, Ryten M, Forabosco P, Schorge S, Hersheson J, Hardy J, Houlden H. United Kingdom Brain Expression C. Insights from cerebellar transcriptomic analysis into the pathogenesis of ataxia. JAMA Neurol 2014;71:831-9.

5 Smeets CJ, Verbeek DS. Cerebellar ataxia and functional genomics: identifying the routes to cerebellar neurodegeneration. Biochim Biophys Acta 2014;1842:2030-8.

6 Köhler S, Vasilevsky NA, Engelstad M, Foster E, McMurry J, Aymé S, Baynam G, Bello SM, Boerkoel CF, Boycott KM, Brudno M, Buske OJ, Chinnery PF, Cipriani V, Connell $L E$, Dawkins HJ, DeMare LE, Devereau AD, de Vries BB, Firth HV, Freson K, Greene D, Hamosh A, Helbig I, Hum C, Jähn JA, James R, Krause R, F Laulederkind SJ, Lochmüller H, Lyon GJ, Ogishima S, Olry A, Ouwehand WH, Pontikos N, Rath A, Schaefer F, Scott RH, Segal M, Sergouniotis PI, Sever R, Smith CL, Straub V, Thompson R, Turner C, Turro E, Veltman MW, Vulliamy T, Yu J, von Ziegenweidt J, Zankl A, Züchner S, Zemojtel T, Jacobsen JO, Groza T, Smedley D, Mungall CJ, Haendel M, Robinson PN. The human phenotype ontology in 2017. Nucleic Acids Res 2017;45:D865-76.

7 Reimand J, Arak T. Vilo J. g:Profiler-a web server for functional interpretation of gene lists (2011 update). Nucleic Acids Res 2011;15.

8 Montojo J, Zuberi K, Rodriguez H, Bader GD, Morris Q. GeneMANIA: fast gene network construction and function prediction for Cytoscape. F1000Res 2014;3:153.

9 Keshava Prasad TS, Goel R, Kandasamy K, Keerthikumar S, Kumar S, Mathivanan S, Telikicherla D, Raju R, Shafreen B, Venugopal A, Balakrishnan L, Marimuthu A, Banerjee S, Somanathan DS, Sebastian A, Rani S, Ray S, Harrys Kishore CJ, Kanth S, Ahmed M, Kashyap MK, Mohmood R, Ramachandra YL, Krishna V, Rahiman BA, Mohan S, Ranganathan P, Ramabadran S, Chaerkady R, Pandey A. Human Protein Reference Database--2009 update. Nucleic Acids Res 2009:37:D767-72.

10 Chatr-Aryamontri A, Oughtred R, Boucher L, Rust J, Chang C, Kolas NK, O'Donnell L, Oster S, Theesfeld C, Sellam A, Stark C, Breitkreutz BJ, Dolinski K, Tyers M. The BioGRID interaction database: 2017 update. Nucleic Acids Res 2017;45:D369-79.

11 Killcoyne S, Carter GW, Smith J, Boyle J. Cytoscape: a community-based framework for network modeling. Methods Mol Biol 2009;563:219-39.

12 Sama IE, Huynen MA. Measuring the physical cohesiveness of proteins using physical interaction enrichment. Bioinformatics 2010;26:2737-43.

13 Oortveld MA, Keerthikumar S, Oti M, Nijhof B, Fernandes AC, Kochinke K, CastellsNobau A, van Engelen E, Ellenkamp T, Eshuis L, Galy A, van Bokhoven H, Habermann $B$, Brunner HG, Zweier C, Verstreken P, Huynen MA, Schenck A. Human intellectual disability genes form conserved functional modules in Drosophila. PLoS Genet 2013:9:e1003911.

14 Kochinke K, Zweier C, Nijhof B, Fenckova M, Cizek P, Honti F, Keerthikumar S, Oortveld MA, Kleefstra T, Kramer JM, Webber C, Huynen MA, Schenck A. Systematic phenomics analysis deconvolutes genes mutated in intellectual disability into biologically coherent modules. Am J Hum Genet 2016;98:149-64.

15 Robinson MD, McCarthy DJ, Smyth GK. edgeR: a Bioconductor package for differentia expression analysis of digital gene expression data. Bioinformatics 2010;26:139-40.

16 Ritchie ME, Phipson B, Wu D, Hu Y, Law CW, Shi W, Smyth GK. limma powers differential expression analyses for RNA-sequencing and microarray studies. Nucleic Acids Res 2015:43:e47.

17 Matilla-Dueñas A, Sánchez I, Corral-Juan M, Dávalos A, Alvarez R, Latorre P. Cellular and molecular pathways triggering neurodegeneration in the spinocerebellar ataxias. Cerebellum 2010;9:148-66.

18 Crespo-Barreto J, Fryer JD, Shaw CA, Orr HT, Zoghbi HY. Partial loss of ataxin-1 function contributes to transcriptional dysregulation in spinocerebellar ataxia type 1 pathogenesis. PLoS Genet 2010;6:e1001021.

19 Huang S, Yang S, Guo J, Yan S, Gaertig MA, Li S, Li XJ. Large Polyglutamine Repeats Cause Muscle Degeneration in SCA17 Mice. Cell Rep 2015;13:196-208.

20 Huang S, Ling JJ, Yang S, Li XJ, Li S. Neuronal expression of TATA box-binding protein containing expanded polyglutamine in knock-in mice reduces chaperone protein response by impairing the function of nuclear factor-Y transcription factor. Brain 2011;134(Pt 7):1943-58.

21 Meera P, Pulst SM, Otis TS. Cellular and circuit mechanisms underlying spinocerebellar ataxias. J Physiol 2016;594:4653-60.

22 Raman IM, Bean BP. Properties of sodium currents and action potential firing in isolated cerebellar Purkinje neurons. Ann N Y Acad Sci 1999;868:93-6.

23 Burroughs A, Wise AK, Xiao J, Houghton C, Tang T, Suh CY, Lang EJ, Apps R, Cerminara $\mathrm{NL}$. The dynamic relationship between cerebellar Purkinje cell simple spikes and the spikelet number of complex spikes. J Physiol 2017:595:283-99.

24 Mark MD, Schwitalla JC, Groemmke M, Herlitze S. Keeping Our Calcium in Balance to Maintain Our Balance. Biochem Biophys Res Commun 2017;483:1040-50 
25 Liu J, Tang TS, Tu H, Nelson O, Herndon E, Huynh DP, Pulst SM, Bezprozvanny I. Deranged calcium signaling and neurodegeneration in spinocerebellar ataxia type 2.J Neurosci 2009;29:9148-62.

26 Shakkottai VG, Chou CH, Oddo S, Sailer CA, Knaus HG, Gutman GA, Barish ME, LaFerla FM, Chandy KG. Enhanced neuronal excitability in the absence of neurodegeneration induces cerebellar ataxia. J Clin Invest 2004;113:582-90.

27 Kaufman RJ, Malhotra JD. Calcium trafficking integrates endoplasmic reticulum function with mitochondrial bioenergetics. Biochim Biophys Acta 2014;1843:2233-9.

28 Gleichmann M, Mattson MP. Neuronal calcium homeostasis and dysregulation. Antioxid Redox Signal 2011;14:1261-73.

29 Jeppesen DK, Bohr VA, Stevnsner T. DNA repair deficiency in neurodegeneration. Prog Neurobiol 2011;94:166-200.

30 Duan Y, Gross RA, Sheu SS. Ca2+-dependent generation of mitochondrial reactive oxygen species serves as a signal for poly(ADP-ribose) polymerase-1 activation during glutamate excitotoxicity. J Physiol 2007;585(Pt 3):741-58.
31 Narciso L, Parlanti E, Racaniello M, Simonelli V, Cardinale A, Merlo D, Dogliotti E. The response to oxidative DNA damage in neurons: mechanisms and disease. Neural Plast 2016;2016:1-14.

32 Akbari M, Morevati M, Croteau D, Bohr VA. The role of DNA base excision repair in brain homeostasis and disease. DNA Repair 2015;32:172-9.

33 Rulten SL, Caldecott KW. DNA strand break repair and neurodegeneration. DNA Repair 2013;12:558-67.

34 Gilmore EC. DNA repair abnormalities leading to ataxia: shared neurological phenotypes and risk factors. Neurogenetics 2014;15:217-28.

35 Madabhushi R, Pan L, Tsai LH. DNA damage and its links to neurodegeneration. Neuron 2014;83:266-82

36 Shiloh Y, Ziv Y. The ATM protein kinase: regulating the cellular response to genotoxic stress, and more. Nat Rev Mol Cell Biol 2013;14:197-210.

37 Bottini AR, Gatti RA, Wirenfeldt M, Vinters HV. Heterotopic Purkinje cells in ataxiatelangiectasia. Neuropathology 2012;32:23-9.

38 Guo Z, Kozlov S, Lavin MF, Person MD, Paull TT. ATM activation by oxidative stress. Science 2010;330:517-21. 